\title{
New era of personalised epilepsy management
}

\author{
The trial and error approach to epilepsy treatment has not changed for over a century but machine \\ learning and patient derived stem cells promise a personalised and more effective strategy, argue \\ Patrick Kwan and colleagues
}

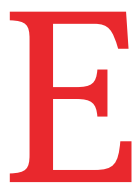
pilepsy affects 50 million people worldwide with no age, ethnic, or geographical boundary. ${ }^{1}$ Patients have recurrent seizures that can lead to injuries, cognitive decline, psychosocial dysfunction, and even death. Epilepsy is caused by brain insults such as trauma, stroke, tumour, inflammation, and infection as well as systemic changes resulting from genomic variation. Patients with epilepsy have increased comorbidities, including cerebrovascular, neurocognitive, and psychiatric diseases. ${ }^{2}$ Better epilepsy control will therefore improve overall brain health.

\section{Uncertainty in treatment response is major problem}

Numerous drugs are available to treat epilepsy as well as non-drug interventions such as resective surgery, neuromodulation, and dietary therapies. Yet the current standard of care still relies on a trial and error approach of sequential regimens of antiseizure medications. Although there are guidelines on drug selection based on broad seizure type (focal or generalised onset) many drugs have similar efficacy when analysed on a group basis. ${ }^{3}$ For any given patient, it is impossible to predict which drug will be most effective and should be selected as the initial treatment. Nor are there surrogate biomarkers that can reliably predict treatment responses or risk of drug resistant epilepsy in the routine

\section{KEY MESSAGES}

- For more than a century the approach to epilepsy treatment has been trial and error because there is no reliable way to predict which medications will work

- Advances in machine learning promise more accurate models to predict treatment outcomes for individual patients

- Genome-wide screening and sophisticated disease models using patient derived stem cells may allow precision epilepsy treatment in future clinical setting. The upshot is that patients must simply wait and see whether their epilepsy will be controlled, usually defined as an absence of seizures for at least one year. ${ }^{4}$ Despite an explosion of new drugs, with over 20 on the market, antiseizure medications fail to control seizures in one third of patients. $^{5}$

Across much of the world most patients newly diagnosed with epilepsy are treated by primary care physicians (box 1). If seizure control is not achieved with initial treatment, patients are referred to a general neurologist who, if further drug treatments fail, then refers them to an epilepsy centre. This sequential care pathway means critical time is lost before patients who may be at high risk of drug resistant epilepsy can be assessed by epilepsy specialists. ${ }^{6}$ Other treatment options, such as surgery, are widely considered a last resort. Sadly, the associated time delay means such treatments may be less effective. ${ }^{7}$ The result is often years of reduced quality of life, lost productivity, and increased mortality. ${ }^{9}$

This predicament might be solved by a reliable method to find patterns linking treatment outcomes to a patient's personal characteristics. Patients with high risk of drug resistant epilepsy could be triaged early, expediting access to the precious resource of specialist care. Recent advances in artificial intelligence (AI) and stem cell research are raising hopes that personalised epilepsy management could soon be a viable alternative to this sequential treatment pathway (fig 1).

\section{Medical artificial intelligence}

Recent advances in machine learning, a subset of AI, offer novel ways to develop prediction models that are more accurate than traditional statistical modelling. Machine learning is being explored in epilepsy to forecast and detect seizures through recognition of electroencephalography (EEG) patterns. A recent study used 9571 routinely collected scalp EEG records to train a deep neural network that outperformed experts in detecting interictal epileptiform discharges. ${ }^{11}$ Researchers have also used time series based algorithms (for example, the line length algorithm used in responsive neurostimulation systems ${ }^{12}$ ) to analyse controlled, continuously acquired, intracranial EEG signals to develop seizure warning systems. ${ }^{13}$ If shown to be effective in large scale clinical trials, such systems could help patients pre-empt and reduce injury from seizures.

Recent studies have used drug dispensing databases to develop models to predict drug treatment responses. ${ }^{1415}$ Although these are large datasets, the models do not capture detailed information about the individual or the disease and therefore lack potentially important data on treatment outcomes. Medical records, on the other hand, include comprehensive clinical information on epilepsy management and are a fuller repository of factors potentially linked to treatment outcomes.

In the past five years a more advanced subfield of machine learning, called deep learning, has achieved impressive gains in the areas of image recognition, natural language processing, and speech. The superior performance of deep learning over traditional machine learning mainly arises from its depth of architecture and the capacity to scale massive amounts of data and continuously improve with more observations. Extended graphical models have shown superiority in modelling dynamic and complex graph structured data, such as clinical data. These models can unravel the hidden structure and reveal the complex links between clinical variables to derive predicted probabilities of the outcome of interest.

In medical AI, models have been shown to be capable of automatically discovering and learning from complicated "hidden (latent) spaces" by encoding multiple observed features to fewer representation variables that are optimised for predicting the outcome of interest. ${ }^{16-19}$ For instance, graphical models recently identified the spatiotemporal evolution of epileptic seizures by leveraging spatial and temporal information in structural longitudinal data. $^{20}$ 


\section{Box 1: Current treatment practice}

Jane is a 30 year old woman with newly diagnosed temporal lobe epilepsy. Her EEG appears normal but an MRI shows right hippocampal sclerosis, the likely source of her seizures. In line with guidelines, ${ }^{10}$ Jane's general practitioner prescribes lamotrigine, one of the many drugs shown to be effective against focal seizures. Jane has more seizures and a few months later visits her GP who, appropriately, refers her to a general neurologist. Over the next few years Jane tries various drugs, some of which are stopped because of side effects, and is eventually maintained on three drugs. Despite this, ongoing seizures mean Jane cannot drive, loses her job, and becomes depressed. The neurologist refers her to an epilepsy centre to be evaluated for surgery.

Biomedical Bidirectional Encoder Representations from Transformers $(\text { BioBERT })^{21}$ is the latest pretrained biomedical language representation model based on deep learning techniques and designed for biomedical text mining. BioBERT, released in early 2020, supports model training by facilitating use of unstructured data from many additional sources, such as electronic health records and clinical reports. This is combined with powerful deep learning graphical models, allowing researchers to include more granular and potentially useful information in the analysis of treatment outcomes, something not possible with traditional statistical analysis.

These AI advances raise the hope of robust models to predict drug treatment responses. A study at the Stanford Epilepsy Center is developing AI models to predict outcomes of antiseizure medication treatment from participants' seizure, genetic, physical, physiological, medication, and environmental data. ${ }^{22}$ The ideal AI algorithm and input data to predict drug treatment responses are not yet known. Future studies should therefore explore more advanced and complex graphical AI models and use data from large, longitudinal epilepsy registries so that comprehensive information can be mined from patients' medical records. Those studies might enhance the models by applying natural language processing tools to extract unstructured data.

Although clinicians may be getting used to software being incorporated into their workflow, the "black box" nature of deep learning based AI systems could still hamper uptake. There have, however, been recent advances in the visualisation of AI based support of clinical decision making processes across multiple areas. ${ }^{23}$ Determining the interoperability of models that visualise knowledge encoded within deep neural networks, and how this is affected by the data input, is also important for the development of novel treatment models. ${ }^{24}$
Genomics, stem cells, and precision treatment Genome-wide screening of patients has identified a growing list of genes, single nucleotide gene variants (SNVs), and genome hotspots associated with epilepsy. Around $70 \%$ of epilepsy cases may be due to one or more genetic factors. ${ }^{2526}$ Even as examples emerge,${ }^{27} 28$ it remains unclear to what extent identification of pathogenic genetic variants will influence treatment decisions in clinical practice. To address that knowledge gap, a randomised controlled trial is investigating the clinical utility and cost effectiveness of whole genome sequencing in patients with refractory epilepsy. ${ }^{29}$

If genetic knowledge is to translate into better treatment it is critical to have a clearer understanding of the functional role of genetic variation. Researchers have
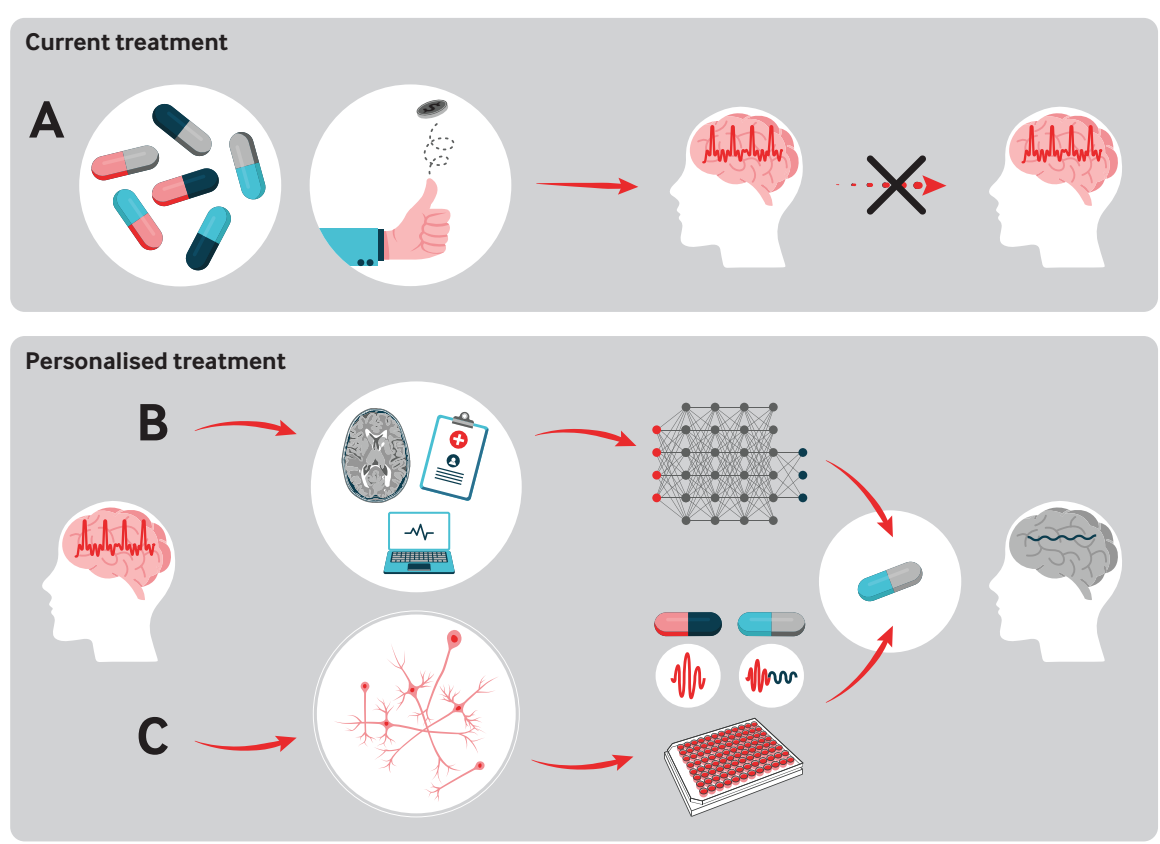

Fig 1 | Simplified conceptual view of how personalised treatment may be applied in epilepsy. Instead of the present trial and error approach (a) physicians could consult the decision supporting software for drug selection and identifying patients with high risk of drug resistance (b). Blood cells are obtained from patients to derive personalised disease models for drug screening to identify targeted and effective treatment (c) 
or "differentiated" into a variety of cell lineages, including multiple neural subtypes (fig 2). These patient derived neural models allow the study of genetic variation for a broad range of neural phenotypes, such as abnormal neural morphology or synaptic transmission, which is not possible with traditional models. The models have been used to identify the abnormal behaviour of neurones that carry highly pathogenic gene variants, as seen in early developmental encephalopathies. $^{33}$

The advantages of iPSC based disease models include the ability to explore the combined effects of multiple SNVs in a single patient and cases where the genetic lesion is unknown. ${ }^{34}$ There are, however, important hurdles to be overcome before these models can be used routinely in clinical decision making. More research is needed to show whether a hyperactive network phenotype, a hallmark of clinical epilepsy, can be reproduced in a dish. More study, too, is required to establish the relation between electrical activity measured in these in vitro models and epileptiform activity observed on EEG.

Current iPSC based neural models lack sufficient cellular complexity to establish seizure-like activity. Researchers are therefore turning to cerebral organoids that contain organised, multicellular tissue structures found in the brain. ${ }^{35}$ More complex disease models will be essential to accurately model dysfunction in the broad range of cell types and brain regions that cause epilepsy in humans. In addition, multielectrode arrays, which record the coordinated interplay of networked neurons, have been used to detect EEG-like signatures from cultured cerebral organoids. ${ }^{36}$

Since iPSC based models can be grown indefinitely without risk to the patient, they will be important for high throughput screening of candidate compounds in patient specific conditions. The aim is to identify novel, targeted antiseizure medications. Indeed, these models have been successfully used for high throughput drug screening in other central nervous system diseases. ${ }^{37}$ Such drug screening platforms could overcome our heavy reliance on traditional rodent models, which has hindered the development of antiseizure medications and helps explain why more than a third of patients with epilepsy lack effective treatment.

\section{Future of personalised epilepsy management}

If personalised epilepsy management is to become a reality technological advances

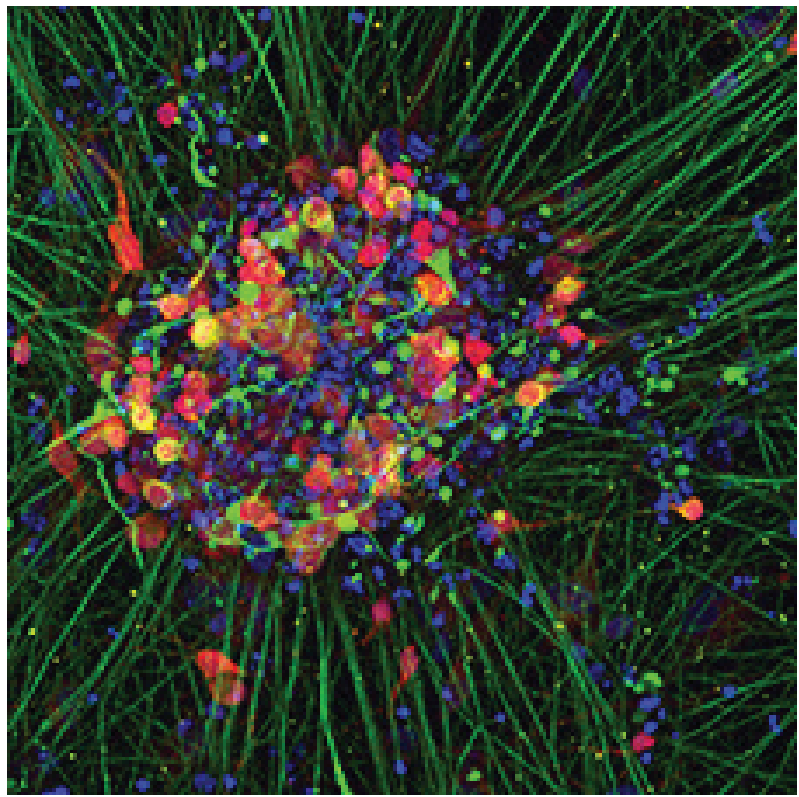

Fig 2 | "Epilepsy in a dish" models comprise human neurons derived from patient iPSCs. Here they grow as small clusters of neurones (red) and from which project long ranging axons (green). The nuclei of supporting astrocytes and senescent cells are shown in blue. These models will advance precision medicine and identification of new targeted drugs for epilepsy

must be coupled with improved health education and access to specialist care. The Australian Epilepsy Project (https:// epilepsyproject.org.au) aims to build a network of centres to improve access for people living with epilepsy, especially in rural regions. It will recruit 8000 patients over five years to develop clinical decision support models by training AI algorithms on advanced imaging, neurocognitive functions, genetics, and other clinical features. The outcome prediction models will not only be of value to specialists but help

\section{Box 2: The near future}

Al based clinical decision support models accurately predict the likely success of each antiseizure medication for an individual patient. The models are converted to software, approved by the US Food and Drug Administration and other regulatory authorities, under the category "software as a medical device." Used standalone or integrated into electronic medical record systems, the software improves performance using real world feedback. It identifies patients at high risk of drug resistant epilepsy and expedites early, targeted access to costly specialised care or surgical evaluation. The software proves cost effective and is used to prioritise patients for access to specialised epilepsy centres.

Jane's GP diagnoses epilepsy and enters Jane's data into the Al based treatment decision support software. The information includes seizure type and frequency, epilepsy risk factors, EEG and MRI results, medical history, and demographic and other relevant data. Within seconds, the software concludes there is an $80 \%$ chance Jane's epilepsy will not respond to the available antiseizure medications and recommends she is prioritised for specialised care. The GP promptly refers Jane to an epilepsy centre. She has a blood sample taken for screening using cerebral organoids against a library of compounds. The screening shows a drug currently used to treat another condition may be effective. Based on a favourable assessment of the risks and benefits, the drug is "repurposed" to treat Jane's epilepsy. Her seizures stop and her life is back on track. 


\section{acteristics can yield predictive models more powerful than those using either data type alone. Our hope is that a convergence of technologies could, within five to 10 years, make personalised epilepsy management a clinical reality.}

We thank science journalist and Monash University academic Paul Biegler for editing and proofreading this article.

Contributors and sources: $\mathrm{ZC}$ is a neuroscientist and biostatistician with expertise in epilepsy outcome research; $B R$ is a cell biologist; $A A B$ is a stem cell biologist; $A A$ is a bioinformatician; $Y M$ is a molecular neuroscientist; TOB is a neurologist and head of the Central Clinical School, Monash University, and programme director of Alfred Brain, and deputy director of research, Alfred Health; ZG is a machine learning scientist and leads Monash Medical Al Group; XW is vice director of neurology, First Affiliated Hospital of Chongqing Medical University; PK is a director of epilepsy, Alfred Health. PK conceived the idea, which arose from a series of discussions about the topic with coauthors. ZC, BR, AAB, ZG, and PK drafted the first manuscript. All the authors critically reviewed the manuscript. PK is the guarantor article.

Competing interests: We have read and understood BMJ policy on declaration of interests and have the following interests to declare: ZC is supported by an early career fellowship from the National Health and Medical Research Council (NHMRC) of Australia (GNT1156444). PK is supported by a Medical Research Future Fund (MRFF) from the NHMRC of Australia (MRF1136427). PK and BR are supported by a MRFF Stem Cell Therapies grant (APP1201781). His institution has received speaker or consultancy fees and/or research grants from Biscayne, Eisai, GW Pharmaceuticals, LivaNova, Novartis, UCB Pharma and Zynerba. TOB is supported by a programme grant from the NHMRC of Australia (APP1091593), and the Royal Melbourne Hospital Neuroscience Foundation. AAB, $A A, Y M, Z G$, and XW report no conflicts of interest.

Provenance and peer review: Commissioned; externally peer reviewed.

This article is part of a series launched at the Chinese Stroke Association annual conference on 10 October 2020, Beijing, China. Open access fees were funded by the National Science and Technology Major Project. The BM/ peer reviewed, edited, and made the decision to publish these articles.

Zhibin Chen, postdoctoral fellow ${ }^{1,2,3}$

Ben Rollo, postdoctoral fellow ${ }^{2}$

Ana Antonic-Baker, postdoctoral fellow ${ }^{2}$

Alison Anderson, postdoctoral fellow ${ }^{2}$

Yuanlin Ma, postdoctoral fellow ${ }^{1}$

Terence J O’Brien, professor ${ }^{2,4}$

Zongyuan $\mathrm{Ge}$, senior research fellow ${ }^{5,6,7}$

Xuefeng Wang, professor $^{1}$

Patrick Kwan, professor $r^{1,2,3,4}$

${ }^{1}$ Chongqing Key Laboratory of Neurology, First Affiliated Hospital, Chongqing Medical University, Chongqing, China

${ }^{2}$ Department of Neuroscience, Central Clinical School, Monash University, Melbourne, Australia

${ }^{3}$ School of Public Health and Preventive Medicine, Monash University, Melbourne, Australia

${ }^{4}$ Department of Neurology, Alfred Hospital, Melbourne, Australia

${ }^{5}$ Faculty of Engineering, Monash University, Melbourne, Australia
${ }^{6}$ eResearch Centre, Monash University, Melbourne, Australia

${ }^{7}$ Airdoc Research Australia, Melbourne, Australia

Correspondence to: P Kwan

patrick.kwan@monash.edu

\section{(c) (1) (8) OPEN ACCESS}

This is an Open Access article distributed in accordance with the Creative Commons Attribution Non Commercial (CC BY-NC 4.0) license, which permits others to distribute, remix, adapt, build upon this work non-commercially, and license their derivative works on different terms, provided the original work is properly cited and the use is non-commercial. See: http://creativecommons.org/ licenses/by-nc/4.0/.

\section{Check for updates}

1 Fiest KM, Sauro KM, Wiebe S, et al. Prevalence and incidence of epilepsy: A systematic review and meta-analysis of international studies. Neurology 2017;88:296-303. doi:10.1212/ WNL.0000000000003509

2 Chen Z, Liew D, Kwan P. Excess mortality and hospitalized morbidity in newly treated epilepsy patients.Neurology 2016;87:718-25. doi:10.1212/ WNL.0000000000002984

3 Perucca E, Brodie MJ, Kwan P, Tomson T. 30 years of second-generation antiseizure medications: impact and future perspectives.Lancet Neurol 2020;19:544-56. doi:10.1016/S1474 4422(20)30035-1

4 Kwan P, Arzimanoglou A, Berg AT, et al. Definition of drug resistant epilepsy: consensus proposal by the ad hoc Task Force of the ILAE Commission on Therapeutic Strategies.Epilepsia 2010;51:1069-77. doi:10.1111/j.1528-1167.2009.02397.x

5 Chen Z, Brodie MJ, Liew D, Kwan P. Treatment outcomes in patients with newly diagnosed epilepsy treated with established and new antiepileptic drugs: a 30-year longitudinal cohort study. JAMA Neurol 2018;75:279-86. doi:10.1001/ jamaneurol.2017.3949

6 Firkin AL, Marco DJ, Saya S, et al. Mind the gap: Multiple events and lengthy delays before presentation with a "first seizure."Epilepsia 2015;56:1534-41. doi:10.1111/ epi.13127

7 Bjellvi J, Olsson I, Malmgren K, Wilbe Ramsay K. Epilepsy duration and seizure outcome in epilepsy surgery: A systematic review and meta-analysis. Neurology 2019;93:e159-66. doi:10.1212/ WNL.0000000000007753

8 Lamberink HJ, Otte WM, Blümcke I, Braun KPJ, European Epilepsy Brain Bank writing group, study group, European Reference Network EpiCARE. Seizure outcome and use of antiepileptic drugs after epilepsy surgery according to histopathological diagnosis: a retrospective multicentre cohort study.Lancet Neurol 2020;19:748-57. doi:10.1016/S14744422(20)30220-9

9 Bell GS, Sinha S, Tisi Jd, et al. Premature mortality in refractory partial epilepsy: does surgical treatment make a difference?) Neurol Neurosurg Psychiatry 2010;81:716-8. doi:10.1136/ jnnp.2008.170837

10 National Institute for Health and Care Excellence. Epilepsies: diagnosis and management: clinical guideline 137. 2012. https://www.nice.org.uk/ guidance/cg137

11 Jing J, Sun H, Kim JA, et al. Development of expertlevel automated detection of epileptiform discharges during electroencephalogram interpretation. JAMA Neurol 2020;77:103-8. doi:10.1001/ jamaneurol.2019.3485
12 Duckrow RB, Tcheng TK. Daily variation in an intracranial EEG feature in humans detected by a responsive neurostimulator system. Epilepsia 2007:48:1614-20. doi:10.1111/j.1528 1167.2007.01091.x

13 Kuhlmann L, Lehnertz K, Richardson MP, Schelter B, Zaveri HP. Seizure prediction - ready for a new eraNat Rev Neurol 2018;14:618-30. doi:10.1038/s41582018-0055-2

14 Delen D, Davazdahemami B, Eryarsoy E, Tomak L, Valluru A. Using predictive analytics to identify drug-resistant epilepsy patients. Health Informatics / 2020;26:449-60. doi:10.1177/1460458219833120

15 Devinsky O, Dilley C, Ozery-Flato M, et al. Changing the approach to treatment choice in epilepsy using big data.Epilepsy Behav 2016;56:32-7 doi:10.1016/i.yebeh.2015.12.039

16 Van Der Maaten L. Accelerating t-SNE using tree-based algorithms. J Mach Learn Res 2014;15:3221-45.

17 Shickel B, Tighe PJ, Bihorac A, Rashidi P. Deep EHR: a survey of recent advances in deep learning techniques for electronic health record (EHR) analysis.IEEE J Biomed Health Inform 2018;22:1589604. doi:10.1109/JBHI.2017.2767063

18 Esteva A, Robicquet A, Ramsundar B, et al. A guide to deep learning in healthcare. Nat Med 2019;25:24-9. doi:10.1038/s41591-018-0316-z

19 Shen D, Wu G, Suk H-I. Deep learning in medical image analysis. Annu Rev Biomed Eng 2017;19:22148. doi:10.1146/annurev-bioeng-071516-044442

20 Covert I, Krishnan B, Najm I, et al. Temporal graph convolutional networks for automatic seizure detection. arXiv 2019:190501375. [Preprint.]

21 Lee J, Yoon W, Kim S, et al. BioBERT: a pretrained biomedical language representation model for biomedical text mining. Bioinformatics 2020;36:1234-40. doi:10.1093/ bioinformatics/btz682

22 Doc.ai. Epilepsy:digital health trial overview. https:// doc.ai/research-trials/epilepsy

23 Tschandl P, Rinner C, Apalla Z, et al. Human-computer collaboration for skin cancer recognition. Nat Med 2020:26:1229-34. doi:10.1038/s41591-0200942-0

24 Ribeiro MT, Singh S, Guestrin C. "Why I trust you?" Explaining the predictions of any classifier. Proceedings of the 22nd ACM SIGKDD international conference on knowledge discovery and data mining, San Francisco, Aug 2016.

25 Thomas RH, Berkovic SF. The hidden genetics of epilepsy-a clinically important new paradigm. Nat Rev Neurol 2014;10:283-92. doi:10.1038/ nrneurol.2014.62

26 Epi25 Collaborative.Ultra-rare genetic variation in the epilepsies: a whole-exome sequencing study of 17,606 individualsAm J Hum Genet 2019;105:267 82. doi:10.1016/j.ajhg.2019.05.020

27 Perucca P, Scheffer IE, Harvey AS, et al. Real-world utility of whole exome sequencing with targeted gene analysis for focal epilepsyEpilepsy Res 2017;131:18. doi:10.1016/j.eplepsyres.2017.02.001

28 Chen P, Lin JJ, Lu CS, et al, Taiwan SJS Consortium. Carbamazepine-induced toxic effects and HLA-B ${ }^{\star} 1502$ screening in TaiwanN Engl f Med 2011;364:1126-33. doi:10.1056/ NEJMoa1009717

29 Clinical utility and cost-effectiveness of immediate vs delayed whole genome sequencing for refractory epilepsy in children and adults: multicentre randomised controlled trial. https:// www.anzctr.org.au/Trial/Registration/TrialReview. aspx?id=375633\&isReview=true

30 Catterall WA. Dravet syndrome: a sodium channel interneuronopathy. Curr Opin Physiol 2018;2:42-50. doi:10.1016/i.cophys.2017.12.007

31 Yu FH, Mantegazza M, Westenbroek RE, et al. Reduced sodium current in GABAergic interneurons in a mouse model of severe myoclonic epilepsy in 
infancy. Nat Neurosci 2006;9:1142-9. doi:10.1038/ nn1754

32 Shi XY, Tomonoh Y, Wang WZ, et al, Epilepsy Genetic Study Group, Japan. Efficacy of antiepileptic drugs for the treatment of Dravet syndrome with different genotypes. Brain Dev 2016;38:40-6. doi:10.1016/j. braindev.2015.06.008

33 Higurashi N, Uchida T, Lossin C, et al. A human Dravet syndrome model from patient induced pluripotent stem cells. Mol Brain 2013;6:19. doi:10.1186/1756-6606-6-19

34 Mertens J, Wang QW, Kim Y, et al, Pharmacogenomics of Bipolar Disorder Study. Differential responses to lithium in hyperexcitable neurons from patients with bipolar disorder Nature 2015:527:95-9. doi:10.1038/nature15526

35 Lancaster MA, Renner M, Martin CA, et al. Cerebral organoids model human brain development and microcephaly. Nature 2013;501:373-9. doi:10.1038/nature 12517

36 Trujillo CA, Gao R, Negraes PD, et al. Complex oscillatory waves emerging from cortical organoids model early human brain network development. Cell Stem Cell 2019;25:558-569.e7. doi:10.1016/j. stem.2019.08.002

37 Kumari D, Swaroop M, Southall N, Huang W, Zheng W, Usdin K. High-throughput screening to identify compounds that increase fragile $X$ mental retardation protein expression in neural stem cells differentiated from fragile $X$ syndrome patientderived induced pluripotent stem cells. Stem Cells Transl Med 2015;4:800-8. doi:10.5966/ sctm.2014-0278

38 Liang M, Li Z, Chen T, Zeng J. Integrative data analysis of multiplatform cancer data with a multimodal deep learning approach. IEEE/ACM Trans Comput Biol Bioinform 2015;12:928-37. doi:10.1109/ TCBB.2014.2377729

Cite this as: $B M / 2020 ; 371: \mathrm{m} 3658$

http://dx.doi.org/10.1136/bmj.m3658 